\title{
Adolescentes y Embarazo
}

\author{
HOSPITAL GENERAL DE NEIVA - 1988
}

Dr. Alberto Moreno Rojas*

\section{"Antes de ser Madre, sé mujer" (J.N.P.F. Jamaica)}

\section{INTRODUCCION}

Es la adolescencia un delicado período de transición entre la infancia y la edad adulta donde empiezan a aparecer las diferentes tendencias ideológicas en busca de un definido patrón de identidad, con sus manifestaciones tan variadas de rebeldía y deseos de libertad, expresados como descontento frente a situaciones familiares o a las normas sociales, lo cual lleva a una alteración de los valores tradicionales vinculados a la sexualidad, el matrimonio y la familia.

La adolescencia entendida como tal, no es un problema; es simplemente un proceso de evolución que conlleva una serie de cambios tanto en la esfera somática como en la síquica, que pueden producir una profunda angustia si no encuentra un entendimiento o una buena comunicación con padres, profesores y demás adultos. Conflicto que puede resolverse de diferentes maneras y dejar como resultado la dificultad para establecer posteriormente relaciones afectivo-sexuales satisfactorias y alteraciones en su desempeño familiar y social.

El adolescente, (del Latín ADOLESCERE: crecer, madurar), es un individuo que se encuentra en una fase peculiar de transición biopsicosocial, caracterizada por transformaciones anatómicas y fisiológicas y en busca de una definición de su papel social condicionado por los patrones culturales de su medio (14). Para unificar criterios y con fines estadísticos, se determinaron las edades límites entre 10 y 19 años (15), según lo acordado por la O.P.S.

\footnotetext{
* Médico Ginecobstetra Hospital General Neiva.
}

Al inicio de la década del 70, Duarte-Contreras manifestaba que el embarazo en adolescentes aumentaba de manera "epidémica", especialmente en las de clase económica menos favorecida debido a la pubertad más temprana, a los matrimonios cada vez a menor edad, a la "explosión de la cópula", a la "nueva libertad sexual" y al número de adolescentes que son aproximadamente el cincuenta por ciento de la población femenina (4), lo que pone de manifiesto que ya, desde varios años atrás, la población adolescente era motivo de preocupación e investigación por los estudiosos.

En su libro: "Madres Solteras Adolescentes", Ana Rico de Alonso dice " . . . las mayores oportunidades de relaciones heterosexuales, un desarrollo puberal o menarca a edades más jóvenes, el afloramiento de una sexualidad más temprana y los contenidos deficientes de la educación sexual impartida por padres y maestros, son algunos de los factores asociados con el ejercicio de una genitalidad precoz para un número creciente de jóvenes, con la experiencia de un embarazo indeseado para muchas de ellas"' (16).

En el estudio realizado por Germán Uriza, comenta: "el embarazo en éste período de la vida va a ser en su gran mayoría indeseado; va a provocar tensiones sicológicas, desajustes familiares profundos, complicados con la falta de apoyo familiar o social y la falta de preparación del personal sanitario responsable de atender a la madre adolescente" (19).

\section{OBJETIVOS}

Con gran frecuencia la literatura médica nos está mostrando todos los problemas que implica la atención de la adolescencia embarazada y quienes trabajamos en un hospital de referencia, como lo es 
el nuestro, lo vemos diariamente a través de nuestras pacientes hospitalarias donde se nos confunde la parte afectiva con lo social y la económica y nos encontramos, la mayoría de las veces, con una paciente agresiva, irritable, descuidada en su persona y con poca o mala comunicación, lo que dificulta el proceso de atención médica.

Para conocer mejor nuestra verdadera situación y con el fin de poder planear, normatizar y ejecutar nuestras conductas frente a éste grupo de adolescentes, en su mayoría "Madres Solteras", la revisión retrospectiva que presento va orientada a todos los médicos y personal paramédico que intervienen en los programas de atención a la población adolescente y, de éste modo, mostrar más a fondo los principales problemas que en este grupo se generan.

\section{MATERIAL Y METODOS}

Basado en la revisión retrospectiva de historias clínicas realizado en el período de un año, comprendido entre el 1o. de junio de 1987 y el 31 de mayo de 1988, donde se atendieron 2.437 pacientes embarazadas en el servicio de Obstetricia en el Hospital General de Neiva, encontramos que aquellas pacientes con edades entre los 14 y los 19 años, sin contar los abortos y los embarazos ectópicos, sumaban 596 pacientes, o sea, el $24.5 \%$ de nuestra población en edad reproductiva, que dieron a luz 596 R.N. y constituyeron un grupo de "adolescentes embarazadas" que, desde el comienzo de la revisión, llamó la atención y se orientó la investigación hacia el conocimiento de si su verdadera problemática en nuestro medio hospitalario es debida a su edad adolescente o si hay otros factores que la desencadenen. Para fines de nuestro trabajo consideramos la adolescencia desde los 10 años, época en que "termina la niñez", hasta los 19 años, cuando se considera que el organismo ha llegado a su total desarrollo.

\section{RESULTADOS}

\section{Edad de las Pacientes}

En el análisis retrospectivo en nuestro grupo investigado encontramos que, las pacientes comprendidas entre los 17 y los 19 años, fueron 452, o sea, el $75.9 \%$ de los casos. La paciente más joven tenía 14 años. El grupo de 15-16 años presentó 120 pacientes para el $20.1 \%$. Cuadro 1 .
CUADRO 1

\section{EDAD MATERNA}

\begin{tabular}{|crc|}
\hline & No. & \% \\
\hline$>15$ & 24 & 4.0 \\
$15-16$ & 120 & 20.1 \\
$17-19$ & 452 & 75.9 \\
\hline Total & 596 & 100.0 \\
\hline
\end{tabular}

\section{Estado Civil}

En el grupo de adolescentes estudiadas se encontró que las solteras eran el $32.7 \%$; las de unión libre el $37.6 \%$ y las casadas el $29.7 \%$; Cuadro 2 .

GUADRO 2

ESTADO CIVIL

\begin{tabular}{|lcc|}
\hline & No. & $\%$ \\
\hline Solteras & 195 & 32.7 \\
Unión libre & 224 & 37.6 \\
Casadas & 177 & 29.7 \\
\hline Total & 596 & 100.0 \\
\hline
\end{tabular}

\section{Dependencia Económica}

Según los datos consignados en la historia social de las pacientes se encuentra que, el $62 \%$, dependen del apoyo familiar, discriminado así: $45 \%$ de la familia de ella y $17 \%$ de la familia de él. Otro grupo informa que su sostenimiento es por parte del esposo o compañero y es el $15 \%$; el grupo de las adolescentes que trabaja para su sostenimiento es del $14 \%$ y un último grupo, sin datos de su sostenimiento es del $9 \%$. Cuadro 3 .

\section{Escolaridad}

Podemos observar que el $30.6 \%$ de las adolescentes investigadas (183 casos), tenían estudios primarios incompletos o ningún estudio y sólo el $2.7 \%$ (17 casos) habían terminado estudios secundarios. Cuadro 4. 
CUADRO 3

\section{DEPENDENCIA ECONOMICA}

\begin{tabular}{|cc|}
\hline & $\%$ \\
\hline De la familia & 62 \\
De él: $17 \%$ & \\
De ella: $45 \%$ & \\
\hline Del esposo o compañero & 15 \\
Trabaja & 14 \\
Sin datos & 9 \\
\hline
\end{tabular}

CUADRO 4

ESCOLARIDAD

\begin{tabular}{|lrr|}
\hline & No. & \multicolumn{1}{c|}{$\%$} \\
\hline Sin estudio alguno & 22 & 3.62 \\
Primaria incompleta & 161 & 27.02 \\
Primaria completa & 155 & 26.12 \\
Secundaria incompleta & 241 & 40.54 \\
Secundaria completa & 17 & 2.70 \\
\hline Total & 596 & 100.00 \\
\hline
\end{tabular}

\section{Paridad}

De las adolescentes estudiadas, en este grupo, podemos ver que 405 pacientes o sea el $67.9 \%$ eran primigrávidas; 127 pacientes o sea el $21.3 \%$ era su segunda gestación. Las restantes, 64 pacientes o sea el $10.7 \%$ eran de tercera y cuarta gestación. Cuadro 5 .

CUADRO 5

\section{PARIDAD}

\begin{tabular}{|crc|}
\hline & No. & $\%$ \\
\hline 1. & 405 & 67.95 \\
2. & 127 & 21.30 \\
3. & 50 & 8.39 \\
4. & 14 & 2.35 \\
\hline Total & 596 & 100.00 \\
\hline
\end{tabular}

\section{Edad de Gestación}

En las pacientes adolescentes investigadas se encontraron 79 casos de Recién Nacidos Prematuros, para un $13.25 \%$; los demás (515 casos) fueron de término y solamente 2 de post-término.

\section{Peso al Nacer}

Los datos obtenidos de los recién nacidos con menos de 2.500 gms, fueron 71 casos para el $11.9 \%$ Pesos entre 2.500 y 3.500 gms, se encontraron 365 R.N., o sea el $61.24 \%$ y, el resto, por encima de $3.500 \mathrm{Gms}$.

\section{Tipo de Parto}

El parto vaginal fue el más frecuente con la cifra de 561 casos, o sea, el $94.13 \%$.

En el grupo de adolescentes solamente se practicaron 35 cesáreas para una incidencia de $5.8 \%$.

\section{Mortalidad Fetal y Neonatal}

En el grupo de adolescentes se encontraron 22 recién nacidos muertos $(3.69 \%)$, distribuidos de acuerdo con la edad materna así: 14 años: $1 ; 15$ años: 3; 16 años: 2; 17 años: 5; 18 años: 6; 19 años: 5 .

De acuerdo con las semanas de gestación de estos R.N. muertos encontramos: con menos de 37 semanas, 11 casos; los demás con 37 y más semanas.

Según el peso al nacer entre los R.N. muertos, encontramos: por debajo de $2.500 \mathrm{gms}$, tenemos 13 casos; entre 2.500 y $3.500 \mathrm{gms}$, hay 6 casos y, el resto, por encima de los 3.500 gms.

No hubo mortalidad materna en el grupo investigado.

\section{CONCLUSIONES}

El grupo de las adolescentes embarazadas constituyó el 24.5\% (596 pacientes) de la población en edad reproductiva atendida en el Hospital General de Neiva. El mayor número correspondió al grupo entre 17 y 19 años de edad, o sea, 452 pacientes $(75.9 \%)$.

El gran grupo de las adolescentes que ingresaron al estudio lo formaron las solteras y las de 
unión libre, con el $70.3 \%$ (419 casos) frente a un $29.7 \%$ (177 casos) de las casadas.

El factor económico es uno de los principales agravantes de nuestras pacientes, pues, en su gran mayoría provienen de estratos socioeconómicos bajos y de las zonas periféricas de la ciudad y otras veces de las áreas rurales.

Debido a los bajos recursos económicos de nuestra población estudiada, la adolescente embarazada se convierte en otra carga más para su ya debilitado presupuesto familiar.

Otro de los problemas de nuestras pacientes adolescentes es el tener que abandonar sus estudios, al encontrarse embarazada, debido al rechazo presentado por las instituciones docentes, por su estado y a la falta de apoyo económico.

Al revisar la paridad de las adolescentes, encontramos que el grupo más numeroso lo constituyen las primigrávidas con el $67.5 \%$ de las pacientes, o sea, 405 casos.

Los recien nacidos prematuros constituyeron el $13.25 \%$ de la población gestante adolescente (79 casos), el resto, $86.7 \%$, fueron de término (517 casos).

Los recién nacidos de bajo peso al nacer, solamente fueron el $11.9 \%$, o sea, 71 casos. Con pesos superiores a los 2.500 gms, y más, se encontró el $88.1 \%$, o sea 525 casos.

El parto por vía natural se presentó en el $94.13 \%$ de los casos, o sea, en 561 pacientes.

Las cesáreas practicadas en las adolescentes fueron 35 , o sea, el $5.8 \%$.

La mortalidad feto-neonatal fue de 22 casos $(3.69 \%)$.

El grupo de los 18 años presentó el mayor número de R.N. muertos, con un total de 6 casos.

Los R.N. prèmaturos muertos fueron en total 11 , o sea el $13.92 \%$ de la muestra examinada.

Los R.N. de bajo peso, muertos, sumaron 13 casos, o sea, el 18,3\%.

No hubo mortalidad materna en el grupo investigado.

\section{COMENTARIOS}

Después de revisar la variada bibliografía del embarazo en las adolescentes y comparar con los resultados obtenidos en la revisión practicada, se puede asegurar que los mayores problemas para las adolescentes embarazadas son los factores de tipo sicológico y social. Esto es debido a que, en un momento dado de su vida, se encuentra nuestra adolescente ante una situación nueva, preocupante, sin la debida preparación, con abandono de su fecundante, la mayoría de las veces y el rechazo de su familia, situaciones éstas que la llevan a un estado de rebeldía frente a su medio ambiente debido a que no encontró la comprensión y el apoyo que buscaba para su estado, convirtiéndose en una paciente de difícil manejo que omite sus citas clínicas, no sigue las dietas prescritas y las instrucciones y a no comunicar los signos de alerta que anuncian las complicaciones de su embarazo (8).

Analizando los informes presentados por otras instituciones hospitalarias (10) (12) (20), nos muestran un porcentaje de pacientes adolescentes similar al encontrado en nuestra revisión y que acudieron en busca de servicios médicos para su atención materna. Esto lleva a pensar que tenemos en nuestros hospitales una población adolescente, en fase activa de reproducción, que corresponde a una cuarta parte de las embarazadas de cualquier edad que solicitan servicios de atención obstétrica.

De acuerdo con las observaciones de DuarteContreras, quien concluye que la adolescente en gestación puede llevar su embarazo a término, con un producto normal, sano, al igual que las gestantes de mayor edad y del mismo nivel socioeconómico (5), es lo que se observa en nuestra serie donde los partos por vía natural fueron la norma predominante $y$, desde luego, tomando las debidas precauciones y cuidados del caso.

Teniendo como pauta los datos anteriores podemos afirmar, como lo hace Duarte-Contreras, que "las normas obstétricas y no la edad de la paciente son los indicadores de la conducta que se debe seguir en la atención del parto”. (Subrayo).

Se comenta en algunos de los diferentes estudios consultados, los resultados relacionados con el bajo peso y la prematurez en los productos de las embarazadas adolescentes, tomándolos como una norma propia de su condición. Otros autores informan en sus series que el bajo peso al nacer fue sólo en el 10\% (1), (18), 11.4\% (9) de los R.N. hijos de 
adolescentes y la prematurez, según otros autores, mostró una incidencia de $9.2 \%$ (1), $12 \%$ (5) y comparándolos con los resultados obtenidos en nuestra revisión, se puede asegurar que, la relación Bajo Peso-Edad Materna, no tiene, en las adolescentes, la importancia que se le ha pretendido dar, al igual que el índice de prematurez en este mismo grupo de pacientes, cifras que son factibles de mejorar aún más, con un control prenatal adecuado.

La mortalidad de los R.N. hijos de adolescentes, para nuestro estudio, fue de 22 casos (3.69\%) distribuidos principalmente entre R.N. Prematuros y R.N. de bajo peso, que comparados con las series de pacientes de mayor edad donde los factores causales más comunes han sido los mismos, encontramos en nuestra muestra una baja incidencia, tal como se detalla en algunos trabajos (5) (7) (20), donde las complicaciones del embarazo, parto y puerperio son similares a las observadas en grupos de mayor edad y de iguales condiciones socioeconómicas.

Conociendo que: 1 . Los principales problemas del embarazo en las adolescentes son fundamentalmente de tipo sicológico, debido a su inmadurez emocional y de tipo social, por los problemas a nivel familiar que esta situación va a desencadenar; 2. Que estos embarazos pueden clasificarse como gestaciones deseadas y gestaciones no deseadas y que tanto las unas como las otras necesitan cuidados especiales y por ello estas pacientes son agrupadas en la consulta como casos de alto riesgo obstétrico (2) (6) (19); 3. Que las consecuencias adversas sobre la salud y los problemas sociales y demográficos condicionados por el embarazo en la adolescencia y el Madre-Solterismo en Colombia, son motivo de preocupación ya que las uniones de mujeres menores de 18 años alargan el período de exposición al embarazo y consecuentemente incrementan las tasas de mortalidad materna y/o perinatal e infantil (3), se hace necesario, para la atención prenatal a las adolescentes, conformar, en estas instituciones, un grupo multidisciplinario con personal médico y paramédico suficientemente preparado para ofrecerles una cuidadosa atención durante su embarazo, parto y puerperio e incluirlas dentro de programas educativos con apoyo de sicólogos, enfermeras, nutricionistas, trabajadoras sociales y planificación familiar, lo que ayudará a disminuir los principales riesgos que presenta el embarazo en las adolescentes (13) (17).

De igual forma, la actitud del médico para encarar la problemática de la adolescente, deberá ser siempre la misma: Escuchar atentamente y con actitud comprensiva, a la paciente, para entenderla en su totalidad y saber ver "más allá" y no quedarse en la superficie de lo que plantea sino evaluar cómo se está produciendo, en general, su desarrollo sicofísico y sexual (11).

\section{RECOMENDACIONES}

Después de haber conocido, a través de este recorrido, a la adolescente y sus principales problemas relacionados con el embarazo y teniendo en mente el principal objetivo de nuestro trabajo para poder llegar a una programación efectiva y práctica que responda a las necesidades de ese grupo poblacional que nos preocupa, debemos emprender una campaña, a todo nivel, donde los protagonistas, los adolescentes, sean los beneficiados y hacerles comprender que las medidas que se están tomando son con el único y exclusivo fin de brindarles protección, orientación y ayuda, que tanto la necesitan.

Los puntos principales sobre los cuales se debe insistir, comprenden:

\section{Educación}

Se debe iniciar desde el hogar, seguir en la enseñanza primaria y luego continuarla en la secundaria o, en su defecto, en los clubes de amas de casa, en los Centros de Salud, en las reuniones comunitarias, en las parroquias y donde se le enseñará a los adolescentes lo relacionado con su sistema reproductor, su funcionamiento, las enfermedades de transmisión sexual y los problemas que implica un embarazo a su edad.

\section{Prevención}

Se debe instruir a todos los adolescentes, activos o no sexualmente, respecto de los diferentes métodos de planificación, los beneficios, los riesgos, su control y, ante todo, hacer énfasis en la responsabilidad sobre su sexualidad.

\section{Control}

La adolescente embarazada se incluirá dentro de un grupo de control prenatal multidisciplinario donde recibirá una atención médica especializada, se dará apoyo emocional ante su nuevo estado, se le indicará una actitud positiva hacia su embarazo, se preparará para el parto y atención del R.N. y se le planeará una vida familiar adecuada. 
La presente revisión pone de manifiesto que, para lograr un buen resultado con las adolescentes embarazadas, se requiere, en gran parte, de buenos servicios obstétricos, sicológicos y sociales y además, muestra la realidad de lo que acontece en la población adolescente en la ciudad de Neiva y regiones vecinas, lo que indica la gran similitud con otras localidades en las mismas condiciones socioeconómicas y culturales.

\section{RESUMEN}

Se revisaron las historias clínicas de 596 pacientes adolescentes embarazadas que fueron atendidas en el Hospital General de Neiva y que constituyeron el $24.5 \%$ de la población de maternas, en el

\section{BIBLIOGRAFIA}

1. BARRIOS, A. J. y Cols. Aspectos perinatales de las adolescentes. Revista Colombiana de Obstetricia y Ginecología Vol. 36, No. 2. Pág. 102, 1985.

2. GARDONA, A. F. Adolescencia. Rev. Col. Obstetricia y Ginecología. Vol. 28, No. 2, Pág. 53. 1977.

3. Diagnóstico de Salud Reproductiva en el País. La sexualidad en la adolescencia. Ministerio de Salud, 1988.

4. DUARTE, C.A. Embarazo en adolescentes de 11 a 15 años. Rev. Col. Obstetricia y Ginecología. Vol. 24, No. 4, Pág. 259, 1973.

5. ¿El embarazo en adolescentes es siempre de alto riesgo? Rev. Col. Obstetricia y Ginecología. Vol. 26, No. 6, Pág. 385, 1975.

6. Factores determinantes del embarazo en adolescentes solteras. Rev. Col. Obstetricia y Ginecología. Vol. 36, No. 5, Pág. 291, 1985.

7. GOMEZ PALACINO, J.A. Obstetricia en adolescentes. Rev. Col. Obstetricia y Ginecología. Vol. 23, No. 4, Pág. 353, 1972.

8. HUFFMAN, J.W. Embarazo en las jóvenes y adolescentes. Ginecología en la infancia y en la adolescencia. Editorial Salvat, 1971.

9. LOPEZ-ESCOBAR, G.; RIAÑO, G. y cols. La embarazada adolescente. Serie de Monografías de CCRP. Vol. 14, 109, Sept. 1979. período de un año, comprendido entre el 1o. de junio de 1987 y el 31 de mayo de 1988.

Encontramos que los factores sicológicos y sociales son los más importantes en la generación de los problemas que afectan a las adolescentes en su estado gestacional.

Se afirma que la relación bajo peso - edad materna y prematurez no tiene la importancia que se le ha pretendido dar con respecto al embarazo de las adolescentes.

Se hacen recomendaciones y se toman como puntos a considerar: Educación, Prevención y Control de los adolescentes, para de ésta manera lograr un mejor resultado con nuestras pacientes.

10. WOOLLEY, L.G. y cols. Algunos aspectos de la atención materna en la Secretaría de Salud Municipal de Cali. Rev. Col. Obstetricia y Ginecología. Vol. 38, No. 3, Pág. 201, 1987.

11. MENDEZ, R.J. Ma. Enfoque actual del adolescente por el ginecólogo. Endocrinología Ginecológica. Ed. Médica Panamericana, 1981.

12. MILLER, M.A.; BROOTEN, D.A. Embarazo en adolescentes. Enfermería Maternoinfantil. Edit. Mcgraw-hill, 1a. Edición, 1987.

13. OLDS, S.B.; LONDON, M.L.; LADEWIG, P.A. La adolescente embarazada. Enfermería Maternoinfantil. Editorial Interamericana. 2a. Edición, 1987.

14. O.P.S. - Primer Seminario Latinoamericano sobre adolescencia. Río de Janeiro, 1977.

15. O.P.S. - Primer Seminario Latinoamericano sobre adolescencia, Buenos Aires, 1978.

16. RICO DE ALONSO, A. Madres Solteras Adolescentes. Plaza y Janés, 1986.

17. SCHOLL, T.O.; MILLER, L.K. Prenatal care adequacy and the outcome of adolescent pregnancy. Obstetric and Ginecology. 69: 312-16, 1987.

18. TOVAR, M.C. y Cols. Estudio comparativo de embarazadas adolescentes vs. embarazadas adultas jóvenes. Rev. Col. Obstetricia y Ginecología. Vol. 38, No. 6, Pág. 436, 1987.

19. URIZA, G. Embarazo en adolescentes. Rev. Col. Obstetricia y Ginecología. Vol. 34, No. 2, 1983.

20. URIZA, G.; BARRAGAN, J. Mortalidad Perinatal. Rev. Col. Obstetricia y Ginecología. Vol. 37, No. 2, Pág. 97, 1986. 